\title{
Editorial
}

\section{Hacia una economía del posconflicto}

http://dx.doi.org/10.14718/revfinanzpolitecon.2017.9.1.1

\section{Joan Miguel Tejedor Estupiñán*}

Tras cuatro años de negociaciones entre el Gobierno de Colombia y la guerrilla de las Fuerzas Armadas Revolucionarias de Colombia-Ejército del Pueblo (FARC-EP), desde La Habana, el 24 de agosto de 2016, representantes de Cuba y Noruega, países garantes, junto con las partes negociadoras divulgaron un comunicado conjunto en el cual anunciaban a los colombianos y al mundo entero el fin de un conflicto de más de seis décadas y haber llegado a un "Acuerdo final, integral y definitivo, sobre la totalidad de los puntos de la agenda del Acuerdo General para la Terminación del Conflicto y la Construcción de una Paz Estable y Duradera en Colombia" ${ }^{1}$. Esta Acuerdo fue ratificado en el Congreso de la República y luego llevado a plebiscito el 2 de octubre de 2016 para que los colombianos decidieran la aprobación final de cara a su implementación (Gobierno Nacional y FARC-EP, 2016).

La pregunta formulada a los colombianos en el plebiscito fue: "¿Apoya usted el acuerdo final para la terminación del conflicto y la construcción de una paz estable y duradera?". Los resultados mostraron que de casi 34,9 millones de personas habilitadas para votar, solo poco más de 13 millones $(37,46 \%)$ participaron de esta contienda electoral, en la que el 49,78\% de electores apoyó el sí y un $50,21 \%$ apoyó el NO.

Este estrecho margen de triunfo de quienes representaban la oposición de lo pactado en La Habana también dejó un claro resultado: una derrota política para la gestión e imagen del presidente Juan Manuel Santos Calderón y muchas dudas sobre la legitimidad del sistema democrático en Colombia. En medio de una campaña llena de controversias y en un tarjetón que tenía solo dos respuestas, 86.000 personas no marcaron el voto, otras 170.000 lo anularon, y más preocupante aún fue la abstención de más de 30 millones de personas habilitadas para votar. Además, los análisis regionales mostraron que gran parte de las zonas en conflicto votaron por el sí al Acuerdo, en tanto las zonas urbanas lo hicieron por el NO (Registraduría Nacional del Estado Civil, 2016).

Luego de varias tensiones entre las partes, estos resultados llevaron a reanudar las conversaciones, pero ahora dando cabida a la parte opositora, con el objetivo de llegar a un nuevo acuerdo en el que se replantearan algunos puntos, pues la oposición argumentaba un riesgo para la institucionalidad democrática colombiana por los beneficios que se les otorgarían a las FARC-EP en un proceso con poca legitimidad (Centro Democrático, 2015), aun cuando se consideraba que este Acuerdo, inspirado en

* Magíster en Derechos Humanos y Economista. Editor de la revista Finanzas y Política Económica de la Universidad Católica de Colombia. Dirección de correspondencia: Facultad de Economía, Universidad Católica de Colombia, Carrera 13 N.²7-49 (Bogotá, Colombia). Correo electrónico: jmtejedor@ucatólica.edu.co.

1 Los seis puntos negociados en el acuerdo comprendían: 1) reforma rural integral, 2) participación política, 3) cese al fuego y hostilidades bilateral y definitivo, 4) solución al problema de las drogas ilícitas, 5) víctimas y 6) mecanismos de implementación y verificación (Gobierno Nacional y FARC-EP, 2016). 
procesos de paz como el de Irlanda y Sudáfrica, era uno de los más completos. A pesar de la derrota política en el plebiscito, la comunidad internacional decidió otorgar el Premio Nobel de la Paz al presidente Santos, por su gestión en este proceso y como motivación y apoyo al pueblo colombiano para seguir en la búsqueda de este logro trascendental.

Posteriormente, después de casi dos meses de reuniones de esta nueva Mesa de Negociación, el 22 de noviembre de 2016 se presentó como resultado un nuevo Acuerdo; sin embargo, hubo incertidumbre frente a la postura de la oposición frente a este, a pesar de que se incluyó la mayoría de sus propuestas. A ello se suma la preocupación de los diferentes sectores sociales, que por medio de movilizaciones - tanto en las principales ciudades del país como por redes sociales- después del plebiscito dejaron evidenciada la necesidad de lograr de manera urgente un Acuerdo de Paz Integral (Oficina del Alto Comisionado para la Paz, 2016).

Finalmente, el 30 de noviembre de 2016, luego de cuarenta días de negociación, la mayoría de miembros del Congreso refrendó el nuevo Acuerdo presentado por el Gobierno (Gobierno de Colombia y FARC, 2016), en medio de calurosos debates en los cuales la oposición seguía manifestando su descontento, a pesar de que casi la totalidad de sus propuestas fueron incorporadas (Oficina del Alto Comisionado para la Paz, 2016).

En este contexto, es muy claro que la paz depende hoy en día de la forma en que las instituciones, las corporaciones y los ciudadanos que conforman el Estado colombiano asuman de manera integral dos aspectos principales: en primer lugar, la transformación y evolución de una cultura caracterizada por la violencia y la violación de los derechos humanos², a raíz del conflicto interno entre el Estado colombiano y las FARC-EP, el Ejército de Liberación Nacional (ELN), el Ejército Popular de Liberación (EPL) y las bandas criminales; y en segundo lugar, la superación de la brecha de desarrollo económico y social con respecto a los países desarrollados y sus principales consecuencias: altos niveles de corrupción, desindustrialización, vulneración de los derechos humanos (laborales, sociales, culturales, etc.) y altos niveles de desigualdad. Lo anterior y los retos que representan la globalización y el cambio climático simbolizan los puntos centrales de la elaboración de un pacto nacional que permita alcanzar una paz integral, estable y duradera para Colombia, que no se enfoque solo en el problema social de la violencia, sino que también dé cabida a los problemas estructurales de la economía, la sociedad y el territorio colombiano en su totalidad.

\section{$* * *$}

Luego de la anterior reflexión, desde la revista Finanzas y Política Económica queremos seguir consolidando alternativas desde la ciencia para la solución de estos trascendentales retos; por ello, desde este editorial quiero expresar mis más sinceros agradecimientos a todos los integrantes del equipo de la

2 A pesar de la firma del Acuerdo, en Colombia se siguen asesinando líderes sindicales y políticos, a lo cual se aúnan problemas culturales más profundos que afectan directamente la integridad de los niños, las mujeres, los campesinos y las comunidades indígenas del país. 
Revista: a los integrantes del Comité Editorial y el Comité Científico, al equipo editorial de la Universidad, a nuestros evaluadores y lectores, y, en especial, a los autores de esta y las anteriores ediciones, quienes con su contribución hacen realidad este proyecto y permiten que sea una de la fuentes emergentes de consulta de las ciencias económicas en la literatura científica colombiana e internacional, la cual brinda libre acceso a nuestros públicos de interés ${ }^{3}$.

En este sentido, tengo el honor de presentar las siguientes investigaciones, que, luego de desarrollar un proceso de evaluación caracterizado por el debate científico de retroalimentación entre evaluadores y autores, han sido seleccionadas por su aporte e impacto al conocimiento y la comprensión de los problemas económicos vigentes. En primer lugar, el artículo "Unsustainable Public Debt in a European Fiscal Union?", del profesor Gábor Kutasi, de la Universidad Corvinus de Budapest (Hungría); en segundo lugar, "Marketing + internet = e-commerce: oportunidades y desafíos", de Pedro Barrientos Felipa, profesor de la Universidad Nacional Mayor de San Marcos (Perú); el tercer artículo, "Efectos del desarrollo financiero sobre el crecimiento económico de Colombia y Chile, 1982-2014", de Armando Lenin Támara Ayús, Lina María Eusse Ossa y Andrés Castellón Pérez, profesores de la Universidad EAFIT; en cuarto lugar, "Tendencias del empleo temporal en la industria boyacense, 2000-2013", de los profesores Edilberto Rodríguez Araújo y Lina Marleny López Sánchez, de la Universidad Pedagógica y Tecnológica de Colombia; en quinto lugar, "(Re)discutir al federalismo fiscal como instrumento de desarrollo social en la República Argentina", de Luciano Rezoagli y Cammarata Marín, de la Universidad Nacional del Litoral (Argentina); el sexto documento, "Localización y especialización productiva regional en Colombia", producido por Henry Antonio Mendoza y Jacobo Campo Robledo, profesores de la Universidad Católica

de Colombia; en séptimo lugar lugar, "Relative efficiency within a tax administration: the effects of result improvement", de los profesores Elena Villar Rubio, Pedro Enrique Barrilao González y Juan Delgado Alaminos, de la Universidad de Granada (España); por último, "A comparative review of the role of income inequality in economic crisis theories and its contribution to the financial crisis of 2007-2009", del profesor Thomas Goda, de la Universidad EAFIT (Colombia).

\section{REFERENCIAS}

1. Centro Democrático (2015). Propuesta: Dialogos de paz sin afectar la institucionalidad democrática [comunicado]. Bogotá: Autor. Recuperado de http://www.eltiempo.com/contenido/politica/proceso-de-paz/ ARCHIVO/ARCHIVO-15490235-0.pdf

2. Gobierno Nacional y FARC-EP (2016). Acuerdo Final para la Terminación del Conflicto y la Construcción de una Paz Estable y Duradera (24-08-2016). La Habana: Autor. Recuperado de http://www.urnadecristal. gov.co/sites/default/files/acuerdo-final-habana.pdf.

$3 \quad$ Esto se evidencia en el reconocimiento alcanzado a nivel nacional en los últimos cuatro años, el cual permitió que la revista ascendiera a la categoría A2 de Publindex, y a nivel internacional, al ser reconocida en bases de datos bibliográficas como EconLit, Repec, Econbiz, Scielo, RedALyC, Ebsco, Proquest, ESCI, Index Copernicus y directorios como DOAJ, REDIB, Dialnet y CLASE. 
3. Oficina del Alto Comisionado para la Paz (2016). Sistematización opciones y propuestas voceros del no y lo acordado en el nuevo acuerdo. Bogotá. Recuperado de http://www.altocomisionadoparalapaz.gov.co/procesosy-conversaciones/Documentos compartidos/21-11-2016-CUADRO-Propuestas-y-Ajustes-definitivo.pdf

4. Registraduría Nacional del Estado Civil. (2016). Resultados Plebiscito 2 octubre 2016, República de Colombia. Recuperado de http://plebiscito.registraduria.gov.co/99PL/DPLZZZZZZZZZZZZZZZZZ_L1.htm 